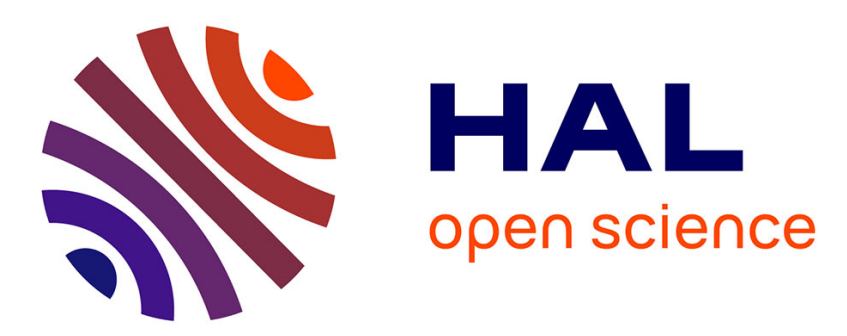

\title{
Une approche multi-échelle de la rhéologie des suspensions concentrées de fibres
}

Steven Le Corre, Pierre J.J. Dumont, Laurent Orgéas, Denis Favier

\section{To cite this version:}

Steven Le Corre, Pierre J.J. Dumont, Laurent Orgéas, Denis Favier. Une approche multi-échelle de la rhéologie des suspensions concentrées de fibres. Revue des composites et des matériaux avancés $=$ Journal of Composite and Advanced Materials, 2005, 15 (3), pp.355-369. 10.3166/rcma.15.355-369 . hal-01007422

\section{HAL Id: hal-01007422 \\ https://hal.science/hal-01007422}

Submitted on 30 Apr 2018

HAL is a multi-disciplinary open access archive for the deposit and dissemination of scientific research documents, whether they are published or not. The documents may come from teaching and research institutions in France or abroad, or from public or private research centers.
L'archive ouverte pluridisciplinaire HAL, est destinée au dépôt et à la diffusion de documents scientifiques de niveau recherche, publiés ou non, émanant des établissements d'enseignement et de recherche français ou étrangers, des laboratoires publics ou privés. 


\section{Une approche multi-échelle de la rhéologie des suspensions concentrées de fibres}

\section{Steven Le Corre* - Pierre Dumont ${ }^{* *}$ - Laurent Orgéas ${ }^{* * *}$ Denis Favier***}

* Institut de Recherche en Génie Civil et Mécanique, CNRS, Ecole Centrale de Nantes 1 rue de la Noё, BP 92101, F-44321 Nantes cedex.

steven.le-corre@ec-nantes.fr

** Laboratoire de Technologie des Composites et Polymères, EPFL, Station 12, CH-1015 Lausanne.

pierre.dumont@epfl.ch

*** Laboratoire Sols-Solides-Structures, CNRS - Universités de Grenoble BP 53, F-38041 Grenoble cedex.

\{laurent.orgeas,denis.favier\}@hmg.inpg.fr

RÉSUMÉ. Ce travail présente une étude de la rhéologie de suspensions de fibres concentrées baignant dans une matrice de type fluide en loi puissance. La méthode d'homogénéisation des structures discrètes est utilisée pour passer de la physique à l'échelle des fibres au comportement macroscopique de la suspension. Cette approche permet de réaliser des expériences de "rhéométrie numérique » sur des volumes élémentaires représentatifs de la suspension afin d'en étudier les propriétés macroscopiques. Les simulations soulignent le rôle prépondérant de l'orientation et de la concentration en fibres, et du comportement de la matrice.

ABSTRACT. The rheology of concentrated fibers in power-law fluids is investigated by upscaling the physics at the fiber scale. The homogenisation method for discrete structures is used to carry out a quantitative study of concentrated fiber suspensions with planar fiber orientation, performing "numerical rheometry experiments" on representative elementary volumes of fiber suspensions. The simulations underline the significant influence of the fiber volume fraction and orientation, as well as the non-Newtonian properties of the suspending fluid on the resulting macroscopic rheological behaviour.

MOTS-CLÉS : suspensions de fibres concentrées, rhéologie, loi puissance, homogénéisation. KEYWORDS: concentrated fiber suspensions, rheology, power-law, homogenisation. 


\section{Introduction}

En raison de leurs bonnes propriétés mécaniques et physico-chimiques, les composites à matrice organique chargée de fibres tels que les " Sheet Molding Compounds » (SMC) ou les Thermoplastiques Renforcés Estampables (TRE) sont très utilisés par l'industrie automobile ou par l'industrie des composants électriques pour fabriquer des pièces semi-structurelles et légères. Leur mise en forme s'effectue par compression dans un moule. Cette étape, où les SMC ainsi que les TRE peuvent être considérés comme des suspensions très concentrées de mèches de fibres de verre dont le fluide suspensif est non newtonien, conditionne très largement les propriétés des produits fabriqués et est encore mal maîtrisée.

La rhéologie des suspensions est pilotée par de nombreux paramètres microstructuraux tels que le taux, l'orientation et la distribution spatiale des fibres, les propriétés mécaniques des fibres et de la matrice et les interactions fibres-matrice et fibre-fibre. A ce jour, de très nombreux travaux ont porté sur le problème de l'étude des suspensions diluées ou semi-diluées dans un fluide newtonien. L'essentiel de ces recherches s'appuie sur les travaux de Jeffery (Jeffery, 1922) et de Batchelor (Batchelor, 1970). Ils conduisent tous à une expression du tenseur des contraintes macroscopiques de la suspension $\boldsymbol{\sigma}^{(s)}$ de la forme :

$$
\boldsymbol{\sigma}^{(s)}=-p^{(s)} \boldsymbol{\delta}+\boldsymbol{\sigma}^{(m)}+\boldsymbol{\sigma}^{(f)},
$$

où la pression $p^{(s)}$ est liée à l'incompressibilité de la suspension, et où $\boldsymbol{\sigma}^{(m)}$ et $\boldsymbol{\sigma}^{(f)}$ sont les contributions de la matrice et des fibres au tenseur des contraintes total. $\boldsymbol{\sigma}^{(f)}$ dépend des interactions fibres-matrice et des interactions fibre-fibre. Ces modèles prennent généralement en compte l'orientation des fibres via des tenseurs d'orientation dont il faut prédire l'évolution (Dupret et al., 1999). Le comportement global de la suspension est alors calculé en couplant un modèle anisotrope pour $\sigma^{(s)}$ et un modèle d'évolution des tenseurs d'orientation durant l'écoulement. Notons enfin que toutes ces approches sont plutôt restreintes au cas de fluides porteurs newtoniens. A notre connaissance, très peu de contributions ont à ce jour porté sur des suspensions semi-diluées de fibres dans une matrice en loi puissance (Goddard, 1976; Gibson et al., 1999).

Les SMC et les TRE sont des suspensions dites concentrées : la distance moyenne entre les mèches de fibres est si faible que les mèches, au cours de la déformation de la suspension, interagissent par des actions à courte distance de type contacts physique et/ou hydrodynamique non newtonien. Pour décrire la rhéologie de ce genre de suspensions, les modèles semi-dilués sont souvent appliqués directement, même s'ils ne prennent pas correctement en compte les mécanismes physiques propres au régime concentré. Les divergences de ces approches avec les viscosités observées expérimentalement (Mackaplow et al., 1996) proviennent essentiellement des interactions entre fibres qui jouent ici un rôle prépondérant. Les études menées sur la nature des interactions entre fibres (Petrich et al., 1998; Ericsson et al., 1997; Servais et al., 1999a; Servais et al., 1999b) tendent à montrer que celles-ci sont une combinaison de frottement 
sec et de frottement hydrodynamique, ce dernier étant lié au cisaillement d'une petite zone de fluide emprisonné $(e)$ au voisinage d'un point de contact.

Dans cette optique, Toll et Månson (Toll et al., 1994) ont proposé un modèle statistique adapté aux suspensions planes de fibres plongées dans un fluide non newtonien. Dans cette approche, les forces d'interactions sont décomposées en une action normale, due à la flexion élastique des fibres selon la normale au plan, et une action tangentielle décomposée en une contribution de frottement sec et une contribution visqueuse non linéaire due au glissement relatif des fibres au niveau d'un point de contact. Servais et al. (Servais et al., 1999b; Servais et al., 1999a) ont plus tard précisé l'expression de cette composante tangentielle. Cette approche est toutefois assez restrictive car elle suppose que la vitesse du centre de chaque fibre est celle de la suspension et que la rotation d'une fibre est une fonction affine du gradient de déformation macroscopique.

Dans le même esprit, Le Corre et al. (Le Corre et al., 2004) ont récemment proposé une approche théorique basée sur la méthode d'homogénéisation des stuctures discrètes périodiques pour décrire le comportement de réseaux de fibres rigides liées par des interactions en loi puissance, ces interactions induisant des forces et des moments locaux dus au mouvement relatif de deux fibres en contact. Ce travail théorique a été ensuite appliqué à la modélisation de la rhéologie des suspensions de type SMC et TRE dans (Le Corre et al., 2005). Pour cela, un modèle micromécanique simple de liaison entre mèches a été proposé et des microstructures fibreuses ont été générées et sollicitées numériquement selon différents chargements mécaniques. Les réponses macroscopiques de microstuctures représentatives ont été comparées avec succès aux résultats expérimentaux de rhéométrie obtenus sur des SMC (Le Corre et al., 2002; Dumont et al., 2003).

Dans cette contribution, on rappelle tout d'abord les hypothèses locales établies dans (Le Corre et al., 2005) pour construire la description de la mécanique à l'échelle des fibres, dans le cas d'une suspension très concentrée de mèches de fibres à orientation plutôt plane. Les résultats théoriques déduits de (Le Corre et al., 2004) ainsi que le schéma numérique de génération et déformation de microstructures utilisés dans (Le Corre et al., 2005) sont ensuite donnés. On analyse alors sur quelques exemples l'impact de la microstructure et du chargement mécanique sur le comportement macroscopique des suspensions étudiées.

\section{Position du problème}

Les suspensions considérées (de type SMC ou GMT) sont des feuilles d'épaisseur $h$ où les mèches de fibres de longueur $l$ sont distribuées de façon homogène dans le plan des feuilles $\mathcal{P} \equiv\left(\boldsymbol{e}_{1}, \boldsymbol{e}_{2}\right)$ et immergées dans un fluide non newtonien. L'épaisseur $h$ (direction $\boldsymbol{e}_{3}$ ) est supposée faible devant $l$. De plus, les suspensions sont supposées incompressibles et suffisamment concentrées pour former un réseau de mèches connectées. Leur déformation est alors pilotée essentiellement par les interactions à 
courte distance entre mèches. Une telle hypothèse est confirmée par de nombreux résultats expérimentaux obtenus sur des SMC et TRE (Ericsson et al., 1997; Servais et al., 1999b; Servais et al., 1999a; Le Corre et al., 2002; Servais et al., 2002; Dumont et al., 2003). Au niveau macroscopique, ceci conduit à négliger $\boldsymbol{\sigma}^{(m)}$ dans (1). Pour évaluer le tenseur des contraintes macroscopique $\boldsymbol{\sigma}^{(f)}$, nous adoptons une méthode de changement d'échelle déterministe (voir partie 3). Comme toute technique d'homogénéisation, les résultats que l'on peut en tirer n'auront de sens que si la condition de séparation des échelles est satisfaite, c'est-à-dire si $\epsilon=d / L \ll 1$, où $d$ et $L$ sont respectivement la longueur caractéristique des hétérogénéités à l'échelle des mèches et celle des pièces fabriquées. Pour les SMC ou les TRE, $d$ est de l'ordre de $l(\approx 25 \mathrm{~mm})$ et $L$ est de l'ordre de $1 \mathrm{~m}$. La séparation des échelles est donc assez bonne puisque $\epsilon \approx 2.510^{-2}$.

\subsection{Géométrie des mèches de fibres}

Les mèches de fibres de verre couramment utilisées dans les SMC sont en général constituées de plusieurs centaines de fibres de verre alignées de $\approx 15 \mu \mathrm{m}$ de diamètre et dont la longueur est de l'ordre de $\approx 25 \mathrm{~mm}$. Elles ont des sections droites plutôt elliptiques très aplaties, où la dimension la plus grande $d_{\max }$ vaut environ $0.6 \mathrm{~mm}$ et où la plus petite $d_{\min }$ vaut environ $0.06 \mathrm{~mm}$. Nous supposerons dans la suite que ces données ne varient pas lors d'une compression. Par ailleurs, comme l'épaisseur des feuilles $h \approx 2-3 \mathrm{~mm}$ est relativement faible par rapport à la longueur des mèches, il est possible considérer que l'orientation moyenne de chaque mèche $b$ définie par le vecteur unitaire $\boldsymbol{e}_{b}$ est contenue dans le plan $\mathcal{P}$. De la même façon, l'aspect très aplati des sections droites permet de supposer que $d_{\max }$ appartient également à ce plan.

\subsection{Cinématique des mèches}

Pour décrire la rhéologie des suspensions considérées, plusieurs hypothèses sur la cinématique, listées ci-dessous, sont formulées.

- En première approximation, de façon analogue à celle adoptée par Servais et al. (Servais et al., 1999a), la flexion des mèches dans $\mathcal{P}$ est négligée et leur section droite est supposée rester elliptique durant la déformation, avec $d_{\max }$ pour grand axe et $d_{\text {min }}$ pour petit axe. Si on note $\tilde{\boldsymbol{x}}$ la projection de $\boldsymbol{x}$ dans $\mathcal{P}$ et $\boldsymbol{G}_{\boldsymbol{b}} \boldsymbol{K}=s_{b} \boldsymbol{e}_{b}+z_{b} \boldsymbol{e}_{3}$, alors la vitesse $\tilde{\boldsymbol{v}}_{b}(K)$ d'un point $K$ situé à l'abscisse curviligne $s_{b}$ et à une hauteur $z_{b}$ du centre de masse $G_{b}$ de la mèche $b$, s'écrit :

$$
\tilde{\boldsymbol{v}}_{b}(K)=\tilde{\boldsymbol{v}}_{b}+s_{b} \omega_{b} \boldsymbol{e}_{3} \times \boldsymbol{e}_{b},
$$

où $\tilde{\boldsymbol{v}}_{b}$ et $\omega_{b}$ sont respectivement la vitesse dans le plan de $G_{b}$ et la vitesse de rotation de la mèche autour de $e_{3}$. 
- Du fait des faibles rapports $d_{\min } / d_{\max }$ et $h / l$, la rotation $\boldsymbol{\omega}_{b}$ d'une mèche $b$ est supposée être contenue dans $\mathcal{P}$ :

$$
\boldsymbol{\omega}_{b}(K) \approx \omega_{b} \boldsymbol{e}_{3}
$$

- Les cas de cisaillement dans l'épaisseur des feuilles ne sont pas étudiés. Si on note $\boldsymbol{L}^{(s)}=\boldsymbol{\nabla} \boldsymbol{v}^{(s)}$ le gradient de vitesse macroscopique de la suspension, seules les situations où $L_{i 3}=L_{3 i}=0(i=1,2)$ sont envisagées.

Ces hypothèses réduisent donc l'étude aux suspensions où les mèches de fibres ont et gardent une orientation plane et se déforment peu dans le plan $\mathcal{P}$, ainsi qu'aux situations où le cisaillement hors plan est faible.

\subsection{Mécanique des interactions entre mèches}

Les constatations expérimentales menées sur des suspensions de fibres concentrées (Ericsson et al., 1997; Servais et al., 1999b; Servais et al., 1999a; Le Corre et al., 2002; Servais et al., 2002; Dumont et al., 2003) fournissent de précieuses indications sur la nature des différents types d'interactions mèche-mèche possibles. En se basant sur ces dernières, il est supposé que les interactions entre mèches sont des forces et des moments de nature visqueuse, dus au cisaillement d'un corps purement visqueux emprisonné $(e)$ au niveau des points de contact, dont la rhéologie serait équivalente à celle d'un fluide homogène visqueux et incompressible en loi puissance occupant le même volume :

$$
\boldsymbol{\sigma}_{(e)}=-p_{(e)} \boldsymbol{\delta}+2 \eta_{(e)}\left(\dot{\gamma}_{(e)} / \dot{\gamma}_{0}\right)^{m-1} \boldsymbol{D}_{(e)},
$$

où $\boldsymbol{\sigma}_{(e)}$ et $p_{(e)}$ sont respectivement le tenseur des contraintes local et la pression dans le fluide emprisonné, $\dot{\gamma}_{(e)}$ est le taux de déformation local, $\eta_{(e)}$ la viscosité du fluide à un taux de déformation caractéristique $\dot{\gamma}_{0}, m$ est l'indice de la loi puissance $(m>0)$, et $\boldsymbol{D}_{(e)}$ est le tenseur vitesse de déformation local.

La géométrie réelle d'une zone d'interaction entre mèches est certainement complexe et difficile à appréhender. Cependant, $d_{\max }$ étant environ 10 fois plus grand que $d_{m i n}$, on peut effectuer une analyse dimensionnelle raisonnable en assimilant cette zone à un prisme d'épaisseur $\alpha$ faible (voir figures 1(b) et 1(c)).

Pendant le déplacement relatif d'un couple $k$ de mèches connectées $b$ et $c$ (le centre de la zone de contact est noté $K$ ), le fluide est soumis à la superposition d'un cisaillement simple, induit par la différence $\triangle \tilde{\boldsymbol{v}}_{k}$ des vitesses des mèches dans le plan, et d'un cisaillement rotatif, dû à la différence des vitesses de rotation des mèches $\triangle \omega_{k} \boldsymbol{e}_{3}$ (voir figure 1(c)). En négligeant les forces $\boldsymbol{f}_{k} \cdot \boldsymbol{e}_{3}$ normales au plan $\mathcal{P}$, (dues principalement à la flexion des mèches hors du plan), i.e. $\boldsymbol{f}_{k} \cong \widetilde{\boldsymbol{f}}_{k}$, il est possible dobtenir des estimations analytiques des forces $\widetilde{\boldsymbol{f}}_{k}$ et moments $M_{k} \boldsymbol{e}_{3}$ de liaisons (Le Corre et al., 2005) :

$$
\tilde{\boldsymbol{f}}_{k}=\frac{\eta_{(e)}}{\alpha} \frac{d_{\max }^{2}}{\left|\sin \triangle \theta_{k}\right|}\left(\frac{\left\|\triangle \tilde{\boldsymbol{v}}_{k}\right\|}{\alpha \dot{\gamma}_{0}}\right)^{m-1} \triangle \tilde{\boldsymbol{v}}_{k}=\mu_{k}\left\|\triangle \tilde{\boldsymbol{v}}_{k}\right\|^{m-1} \triangle \tilde{\boldsymbol{v}}_{k},
$$




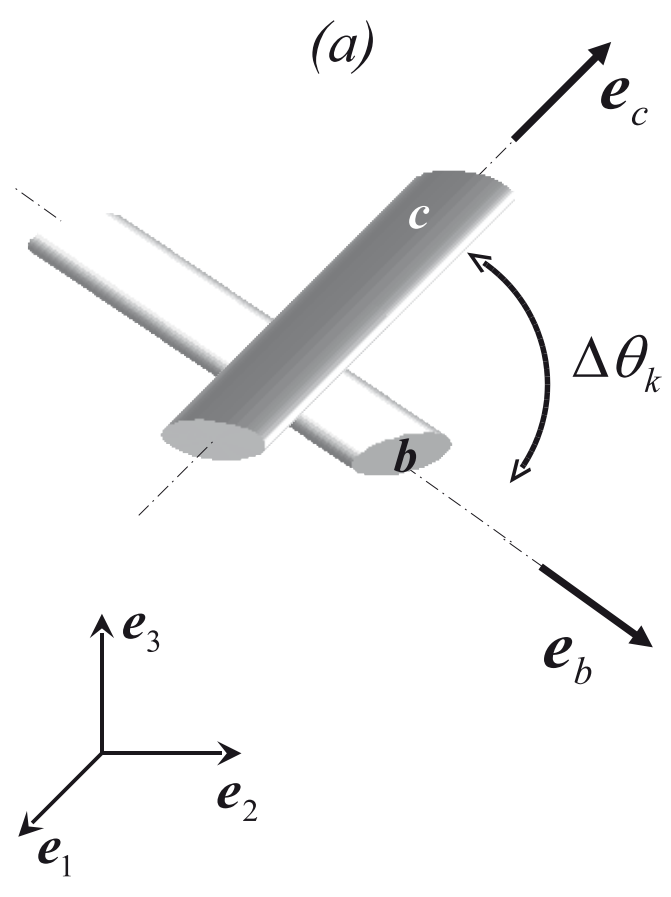

loi puissance
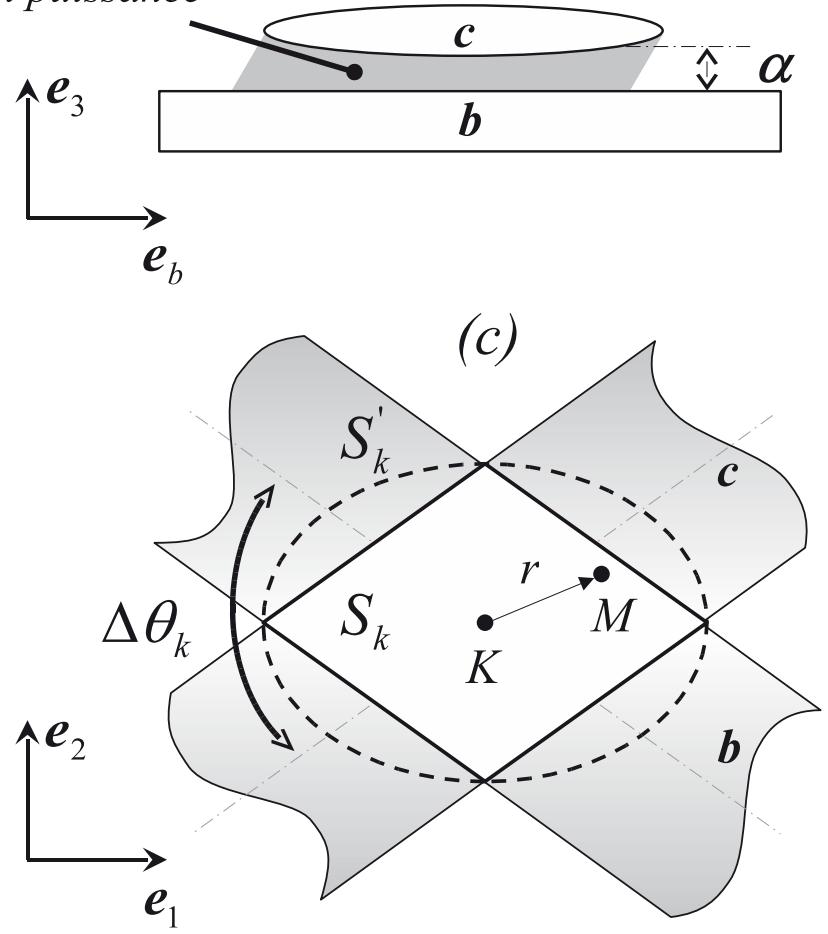

Figure 1. Modélisation des interactions mèche-mèche : vue de côté de la zone cisaillée (b), vue de dessus de la surface d'interaction $(c)$

$$
M_{k}(K)=\mu_{k} \frac{\pi}{m+3}\left(\frac{d_{\max }}{\left|\sin \triangle \theta_{k}\right|}\right)^{m+1}\left|\triangle \omega_{k}\right|^{m-1} \triangle \omega_{k}
$$

\subsection{Equilibre d'une mèche}

En négligeant l'accélération, les forces et les moments volumiques externes, et en introduisant $\mathcal{C}_{b}$ l'ensemble des connections de la mèche $b$, l'équation d'équilibre d'une mèche $b$ peut alors s'écrire (Le Corre et al., 2005) :

$$
\forall b,\left\{\begin{array}{c}
\sum_{k \in \mathcal{C}_{b}} \boldsymbol{f}_{k}=\mathbf{0} \\
\sum_{k \in \mathcal{C}_{b}} M_{k}(K) \boldsymbol{e}_{3}=\sum_{k \in \mathcal{C}_{b}} s_{b} \boldsymbol{f}_{k} \times \boldsymbol{e}_{b}+\sum_{k \in \mathcal{C}_{b}} z_{k} \boldsymbol{f}_{k} \times \boldsymbol{e}_{3}
\end{array}\right.
$$

en notant $\boldsymbol{G}_{\boldsymbol{b}} \boldsymbol{K}_{\boldsymbol{k}}=s_{b} \boldsymbol{e}_{b}+z_{b} \boldsymbol{e}_{3}$. Dans l'équation (7)(b), il est facile de montrer que le second terme du membre de droite est négligeable par rapport au premier $\left(d_{\text {min }} / l=\mathcal{O}\left(\epsilon^{2}\right)\right)$. Ainsi, les forces de contact étant contenues dans $\mathcal{P}$ et fonctions de $\triangle \tilde{\boldsymbol{v}}_{k}$ uniquement, ces équations se réduisent à un problème mécanique bidimensionnel. 


\section{Changement d'échelle}

Pour obtenir le comportement macroscopique d'une telle suspension fibreuse, la méthode d'homogénéisation des structures discrètes périodiques a été utilisée (Moreau et al., 1998; Tollenaere et al., 1998). On rappelle ici les principaux résultats issus de ce travail. Pour plus de détails, le lecteur se référera à (Le Corre et al., 2004). Dans cette méthode, la suspension est vue comme un assemblage périodique de Volumes Elémentaires Représentatifs (VER) contenant chacun $N$ mèches $b$ et $C$ liaisons $k$. Dans l'hypothèse de séparation des échelles, les différents champs $\boldsymbol{u}$ du problème (vitesses de translation et de rotation) sont écrits sous forme de développements asymptotiques « discrets » en puissance de $\epsilon$ :

$$
\widetilde{\boldsymbol{u}}_{b}(\tilde{\boldsymbol{x}})=\widetilde{\boldsymbol{u}}_{b}^{[0]}(\tilde{\boldsymbol{x}})+\epsilon \widetilde{\boldsymbol{u}}_{b}^{[1]}(\tilde{\boldsymbol{x}})+\epsilon^{2} \widetilde{\boldsymbol{u}}_{b}^{[2]}(\tilde{\boldsymbol{x}})+\ldots
$$

Les champs $\tilde{\boldsymbol{u}}_{b}^{[n]}$ sont les fluctuations d'ordre $\epsilon^{n}$ de $\tilde{\boldsymbol{u}}_{b}$, fonctions continues de la variable d'espace $\tilde{\boldsymbol{x}}$ et périodiques par rapport à l'indice $b$. Ces développements asymptotiques sont introduits dans les équations d'équilibre locales, et l'identification des différents termes apparaissant aux mêmes ordres de $\epsilon$ permet d'obtenir les équations d'équilibre et la loi de comportement macroscopique du réseau de fibres considéré. En supposant que les moments de liaisons locaux sont petits devant les moments générés par les forces de liaisons, on montre les différents points suivants.

- Les vitesses au premier ordre $\widetilde{\boldsymbol{v}}_{b}^{[0]}$ ne dépendent pas de la mèche $b$ considérée :

$$
\forall b, \quad \widetilde{\boldsymbol{v}}_{b}^{[0]}=\widetilde{\boldsymbol{v}}^{(s)} .
$$

- Le milieu continu équivalent est un milieu de Cauchy, dont le tenseur des contraintes symétrique s'écrit :

$$
\tilde{\boldsymbol{\sigma}}^{(f)}=\frac{1}{V^{(s)}} \sum_{k=1}^{C} \tilde{\boldsymbol{\xi}}_{k} \otimes \tilde{\boldsymbol{f}}_{k}^{[0]} .
$$

Dans cette équation $V^{(s)}$ est le volume du VER considéré, $\tilde{\boldsymbol{\xi}}_{k}$ est la projection de $G^{b} G^{c}$ dans le plan $\mathcal{P}$ et $\tilde{\boldsymbol{f}}_{k}^{[0]}$ est le premier terme non nul du développement asymptotique des forces d'interaction $\tilde{\boldsymbol{f}}_{k}$. $\widetilde{\boldsymbol{\sigma}}^{(f)}$ peut être déterminé en calculant les forces $\widetilde{\boldsymbol{f}}_{k}^{[0]}$ données par :

$$
\widetilde{\boldsymbol{f}}_{k}^{[0]}=\frac{\eta_{(e)}}{\alpha} \frac{d_{\max }^{2}}{\left|\sin \triangle \theta_{k}\right|}\left(\frac{\left\|\triangle \widetilde{\boldsymbol{v}}_{k}^{[1]}\right\|}{\alpha \dot{\gamma}_{0}}\right)^{m-1} \triangle \widetilde{\boldsymbol{v}}_{k}^{[1]}
$$

avec

$$
\triangle \widetilde{\boldsymbol{v}}_{k}^{[1]}=\widetilde{\boldsymbol{v}}_{c}^{[1]}-\widetilde{\boldsymbol{v}}_{b}^{[1]}+\widetilde{\boldsymbol{L}}^{(s)} \cdot \widetilde{\boldsymbol{\xi}}_{k}+s_{c}^{*} \phi_{c}^{[0]} \boldsymbol{e}_{3} \times \boldsymbol{e}_{c}-s_{b}^{*} \phi_{b}^{[0]} \boldsymbol{e}_{3} \times \boldsymbol{e}_{b}
$$

où $s_{b}^{*}$ désigne la quantité $s_{b} / d$ et $\phi_{b}$ la quantité $L \omega_{b}$. 
- La suspension considérée est un fluide anisotrope en loi puissance, dont l'indice de sensibilité à la vitesse $m$ est égal à celui postulé au niveau des interactions entre mèches.

- Les inconnues cinématiques $\widetilde{\boldsymbol{v}}_{b}^{[1]}$ et $\omega_{b}^{[0]}$ peuvent être calculées en considérant $\widetilde{\boldsymbol{L}}^{(s)}$ comme une donnée dans le problème non linéaire d'auto-équilibre posé sur le VER :

$$
\forall b \in V^{(s)}, \quad\left\{\begin{array}{c}
\sum_{k \in \mathcal{C}_{b}} \tilde{\boldsymbol{f}}_{k}^{[0]}=\mathbf{0} \\
\sum_{k \in \mathcal{C}_{b}} M_{k}^{[0]} \boldsymbol{e}_{3}+\sum_{k \in \mathcal{C}_{b}} s_{b} \boldsymbol{e}_{b} \times \tilde{\boldsymbol{f}}_{k}^{[0]}=\mathbf{0}
\end{array}\right.
$$

avec

$$
M_{k}^{[0]}=\mu_{k}\left[\frac{\pi}{m+3}\left(\frac{d_{\max }}{\left|\sin \triangle \theta_{k}\right|}\right)^{m+1}\right]\left|\omega_{c}^{[0]}-\omega_{b}^{[0]}\right|^{m-1}\left(\omega_{c}^{[0]}-\omega_{b}^{[0]}\right) .
$$

En supposant qu'il n'y a pas de mèches ou de groupes de mèches isolés, on obtient ainsi un problème bien posé qui permet d'obtenir $\widetilde{\boldsymbol{v}}_{b}^{[1]}$ et $\omega_{b}^{[0]}$ quel que soit le taux de déformation macroscopique imposé.

\section{Génération de microstructures représentatives}

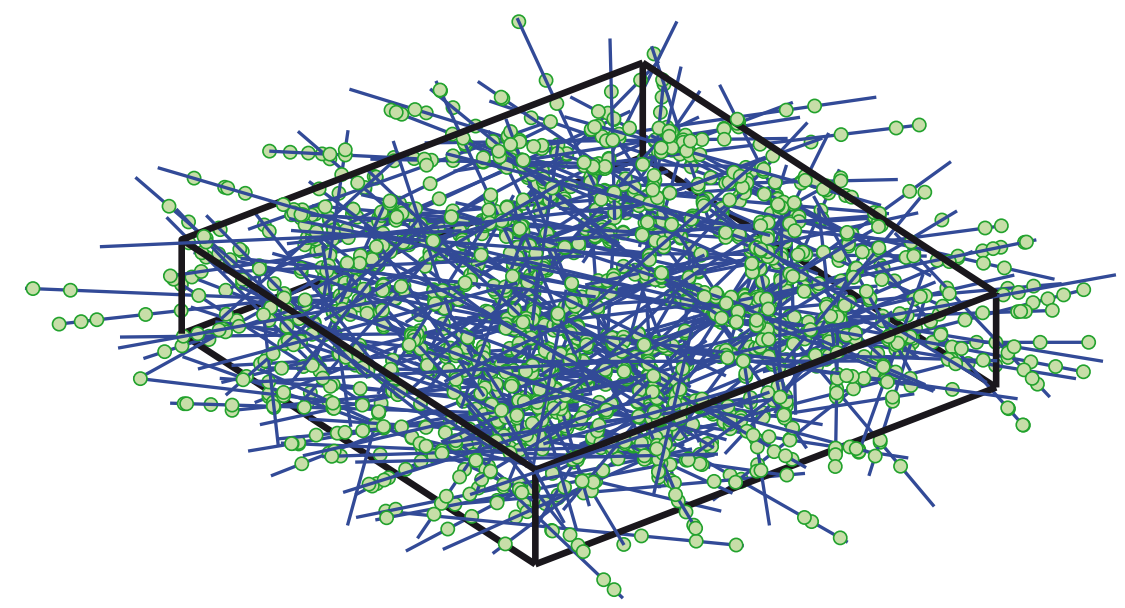

Figure 2. Exemple de VER généré $\left(V^{(s)}=25 \times 25 \times 2.5 \mathrm{~mm}^{3}\right)$, contenant 437 mèches de longueur $25 \mathrm{~mm}\left(f^{(f)}=0.188\right)$. Les symboles $\circ$ représentent la position des connexions $\left(\delta^{*}=3\right)$

Les techniques usuelles d'observation de la morphologie d'un réseau de fibres peuvent fournir des informations sur l'orientation et la position des mèches mais ne permettent pas encore, à notre connaissance, d'obtenir les données relatives à la connectivité du réseau. Le moyen le plus simple s'offrant à nous est donc la génération numérique. 
Pour imiter au mieux et simplement les microstructures réelles, nous générons des réseaux 3D contenant $N$ mèches avec des positions aléatoires $\left(x_{b}, y_{b}\right)$ dans le plan des feuilles comprises entre $[-d / 2, d / 2]$ et des hauteurs aléatoires $z_{b}$ dans $[0, h], d$ et $h$ désignant les dimensions du VER. Différents degrés d'orientation sont ensuite obtenus en utilisant une distribution aléatoire gaussienne des angles $\theta_{b}$ centrée sur $\theta=0$ et avec un écart type variant selon le degré d'orientation souhaité. La connectivité, c'està-dire la position des $C$ contacts entre fibres, est ensuite calculée par une méthode déterministe basée sur une approche de type « tube model » (Ranganathan et al., 1991; Toll, 1993). Pour ce faire, on définit un volume de contrôle $V_{b}$ autour de chaque mèche $b$, et chaque mèche $c$ dont la ligne moyenne intersecte $V_{b}$ est ajoutée à l'ensemble de connectivité de $b, \mathcal{C}_{b} . V_{b}$ est ici une boîte rectangulaire de dimensions $l \times d_{\max } \times$ $\delta^{*} d_{m i n}$, où $\delta^{*}$ est un paramètre sans dimension dont la valeur peut être determinée expérimentalement (Le Corre et al., 2005). Nous fixerons ici sa valeur arbitrairement à 3 . Il est possible de montrer qu'à partir d'un nombre suffisant de mèches dans le VER $(\approx 400)$, les réseaux ainsi générés suivent les prédictions du modèle statistique de Toll (Toll, 1993).

Pour décrire et comparer les différents réseaux de fibres entre eux, nous adopterons ici le tenseur d'orientation d'ordre 2, $\widetilde{A}$ (Advani et al., 1987), plutôt que la fonction de distribution d'orientation (Folgar et al., 1984), plus lourde à manipuler :

$$
\widetilde{\boldsymbol{A}}=\frac{1}{N} \sum_{b=1}^{N} \boldsymbol{e}_{b} \otimes \boldsymbol{e}_{b}
$$

Nous utiliserons également la fraction volumique de mèches $f^{(f)}$, directement liée au nombre de mèches par unité de volume $n^{(f)}$ par

$$
f^{(f)}=\frac{V^{(f)}}{V^{(s)}}=\frac{\pi}{4} l d_{\max } d_{\min } n^{(f)} \quad \text { avec } \quad n^{(f)}=\frac{N}{V^{(s)}} .
$$

Avec ces notations, la densité de connections $c^{(f)}$ s'écrit

$$
c^{(f)}=\frac{C}{V^{(s)}}=\frac{1}{2} n^{(f)} \times n_{c / b},
$$

où le nombre moyen théorique de connexions par mèche $n_{c / b}$ (Toll, 1993) vaut ici :

$$
n_{c / b}=n^{(f)} l \delta^{*}\left(l d_{\min } \phi_{1}+d_{\max } d_{\min } \phi_{2}+d_{\max } d_{\min }\right) .
$$

Dans cette équation, les fonctions d'orientation $\phi_{1}$ and $\phi_{2}$ théoriques (Toll, 1993) sont calculées par

$$
\phi_{1}=\frac{1}{N^{2}} \sum_{b=1}^{N} \sum_{c=1}^{N}\left|\sin \left(\theta_{b}-\theta_{c}\right)\right| \quad \text { et } \quad \phi_{2}=\frac{1}{N^{2}} \sum_{b=1}^{N} \sum_{c=1}^{N}\left|\cos \left(\theta_{b}-\theta_{c}\right)\right| .
$$




\section{Expériences de rhéométrie numérique}

Les VER étant générés et la physique des interactions entre mèches donnée, la rhéologie de ce type de suspension peut maintenant être étudiée via des expériences de rhéométrie numérique dont les différentes étapes sont énumérées ci-dessous.

1) Imposer un gradient de vitesses macroscopique $\widetilde{\boldsymbol{L}}^{(s)}$ dans $\mathcal{P}$. Les suspensions étant supposées incompressibles et comme $L_{i 3}=L_{3 i}=0(i=1,2)$, ceci est équivalent à imposer un gradient de déformation tridimensionnel $\boldsymbol{L}^{(s)}$.

2) Résoudre le système (13) pour calculer les champs $\widetilde{\boldsymbol{v}}_{b}^{[1]}$ et $\omega_{b}^{[0]}$. Ceci est effectué par une méthode itérative de Newton-Raphson.

3) Calculer :

- la dérivée temporelle $\dot{\widetilde{A}}$ du tenseur d'orientation $\widetilde{A}$ définie comme :

$$
\dot{\widetilde{\boldsymbol{A}}}=\frac{1}{N} \sum_{b=1}^{N} \dot{\boldsymbol{e}}_{b} \otimes \boldsymbol{e}_{b}+\boldsymbol{e}_{b} \otimes \dot{\boldsymbol{e}}_{b}=\frac{1}{N} \sum_{b=1}^{N}\left(\boldsymbol{\omega}_{b} \times \boldsymbol{e}_{b}\right) \otimes \boldsymbol{e}_{b}+\boldsymbol{e}_{b} \otimes\left(\boldsymbol{\omega}_{b} \times \boldsymbol{e}_{b}\right)
$$

- le tenseur des contraintes macroscopique $\widetilde{\boldsymbol{\sigma}}^{(f)}$ (dans le cas particulier des réseaux, qui suivent la statistique du modèle tube, une expression intéressante de $\widetilde{\boldsymbol{\sigma}}^{(f)}$ peut être établie en notant $\tilde{\boldsymbol{\xi}}_{k}^{*}=\tilde{\boldsymbol{\xi}}_{k} / l$ :

$\tilde{\boldsymbol{\sigma}}^{(f)}=\eta_{(e)} \frac{\delta^{*}}{\alpha^{m}} \frac{8 f^{(f)^{2}}}{\pi^{2}} \frac{d_{\max }}{d_{\min }}\left(\frac{l}{d_{\max }} \phi_{1}+\phi_{2}+1\right) \frac{1}{C} \sum_{k=1}^{C} \frac{\left\|\triangle \widetilde{\boldsymbol{v}}_{k}^{[1]}\right\|^{m-1}}{\left|\sin \triangle \theta_{k}\right|} \tilde{\boldsymbol{\xi}}_{k}^{*} \otimes \triangle \widetilde{\boldsymbol{v}}_{k}^{[1]}$,

cette équation révèle clairement le rôle de la géométrie des mèches, de la fraction de fibres, de l'orientation $\left(\phi_{1}, \phi_{2}\right)$, de la physique des interactions $\left(\eta_{(e)}, m, \alpha,\left|\sin \triangle \theta_{k}\right|\right)$ et du chargement extérieur $\left(\triangle \widetilde{\boldsymbol{v}}_{k}^{[1]}\left(\widetilde{\boldsymbol{L}}^{(s)}\right)\right)$, sur l'état de contraintes général)

- le tenseur des contraintes macroscopique de la suspension $\boldsymbol{\sigma}^{(s)}$, où une extension $3 \mathrm{D}$ très simple est proposée :

$$
\boldsymbol{\sigma}^{(s)}=-p \boldsymbol{\delta}+\tilde{\boldsymbol{\sigma}}^{(f)} .
$$

Les exemples de simulation présentés dans la suite, qui illustrent l'influence de la microstructure et de la rhéologie des liaisons mèche-mèche sur le comportement de la suspension, ont été réalisés sur des VER ayant les paramètres suivants.

- Les mèches ont pour dimensions $l=25 \mathrm{~mm}, d_{\max }=0.6 \mathrm{~mm}, d_{\min }=$ $0.06 \mathrm{~mm}$, les VER sont des pavés de $25 \times 25 \times 2.5 \mathrm{~mm}^{3}$.

- Les réseaux fibreux ont été générés selon la procédure décrite précédemment, dans les axes du repère $\left(\boldsymbol{e}_{1}, \boldsymbol{e}_{2}\right)\left(\tilde{A}_{12} \approx 0\right)$. L'orientation principale $\tilde{A}_{11}$ étudiée est comprise entre 0 et 1 . Les taux fibres étudiés varient entre 0.05 et 0.3 .

- Deux indices de sensibilité à la vitesse sont envisagés, $m=1$ (comportement newtonien) et $m=0.5$ (comportement rhéofluidifiant). 
- L'épaisseur des zones de contact cisaillées est fixée à $2 \mu m$ (Le Corre et al., 2005).

- Les VER ainsi générés sont déformés sous une sollicitation de type compression en canal, pour laquelle le tenseur de vitesse de déformation est (en $\left.s^{-1}\right)$ :

$$
\boldsymbol{D}^{(s)}=\boldsymbol{e}_{1} \otimes \boldsymbol{e}_{1}-\boldsymbol{e}_{3} \otimes \boldsymbol{e}_{3} .
$$

A sollicitation identique, les niveaux de contraintes obtenus dans les cas $m=1$ et $m=0.5$ sont très différents. Comme seules nous importent ici les tendances obtenues en fonction des paramètres microstructuraux, les réponses en contrainte des différents VER présentées dans les figures suivantes $(3,4$ et 5$)$ ont été normalisées par rapport à un niveau de contrainte de référence $\sigma_{r e f}$. Pour chaque $m$, celui-ci correspond à la composante $\widetilde{\sigma}_{11}^{(f)}$ d'un VER à $f^{(f)}=0.188$ avec une orientation quasi isotrope dans le plan, soumis à une sollicitation de type compression en canal.

\subsection{Influence du taux de fibres}

(a)

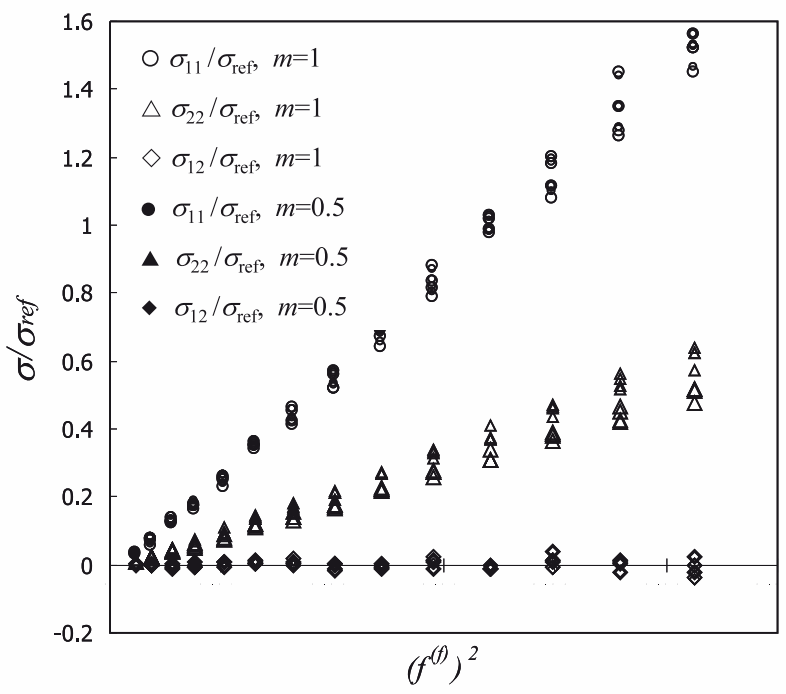

(b)

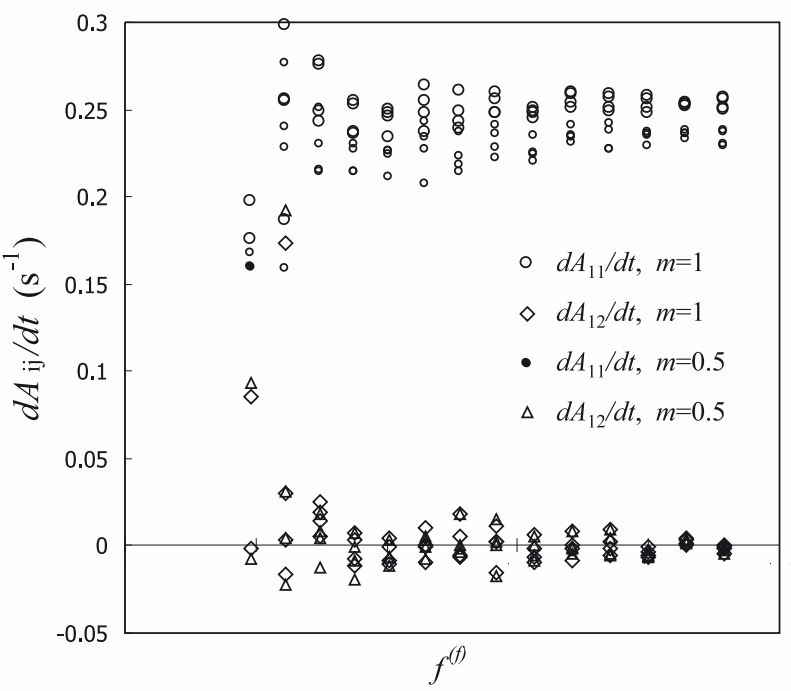

Figure 3. Influence de la fraction de fibres sur la réponse en contraintes $(a)$ et en vitesse d'orientation $(b)$, pour deux indices de sensibilité à la vitesse de déformation ( $m=1$ et $m=0.5)$ - réseaux orientés aléatoirement dans le plan $\left(\widetilde{A}_{11} \approx 0.5\right)$

La figure 3(a) donne l'évolution des contraintes $\widetilde{\sigma}_{i j}^{(f)}$ en fonction du carré du taux de fibres $f^{(f)}$, pour des microstructures quasi isotropes $\left(\tilde{A}_{11} \approx 0.5\right)$ et pour les deux valeurs de $m$ envisagées. Cette figure montre d'une part la forte influence de $f^{(f)}$, et d'autre part que les contraintes sont des fonctions quadratiques de la fraction de fibres, comme cela est suggéré par l'équation (21). Les composantes $\dot{\tilde{A}}_{11}$ et $\dot{\tilde{A}}_{12}$ du tenseur $\dot{\widetilde{A}}$ calculées pour les mêmes microstructures sont reportées sur la figure 3(b). Quelle que soit la microstructure étudiée, on remarque d'une part que les 
composantes $\dot{\tilde{A}}_{12}$ sont très faibles devant les composantes $\dot{\tilde{A}}_{11}$ (essais dans les axes) et d'autre part que le taux de fibres influe très peu sur les résultats. Ces observations sont confirmées par les figures $4(a)$ et $4(b)$ qui représentent les mêmes types de tests sur des microstructures bien orientées dans la direction de la sollicitation $\left(\tilde{A}_{11} \approx 0.8\right)$.

(a)

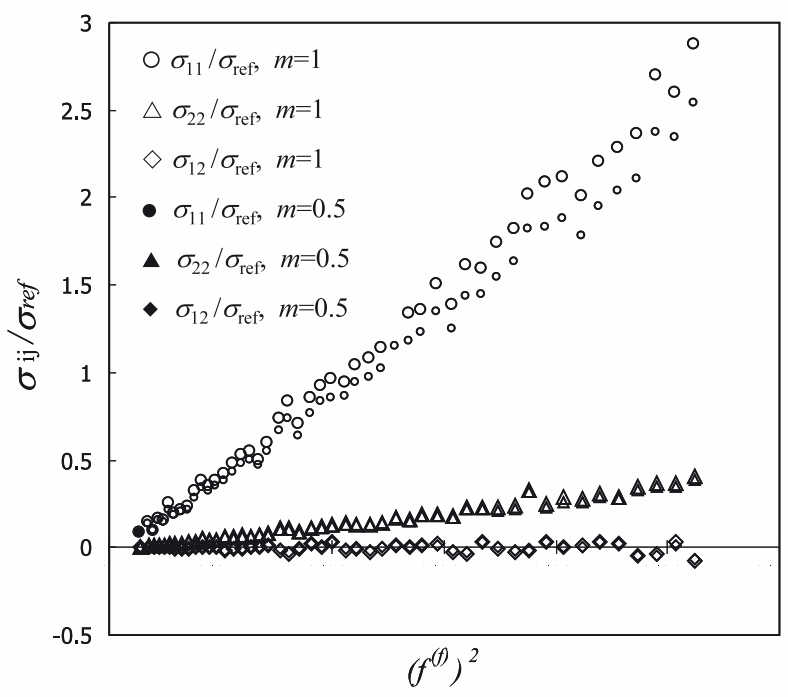

(b)

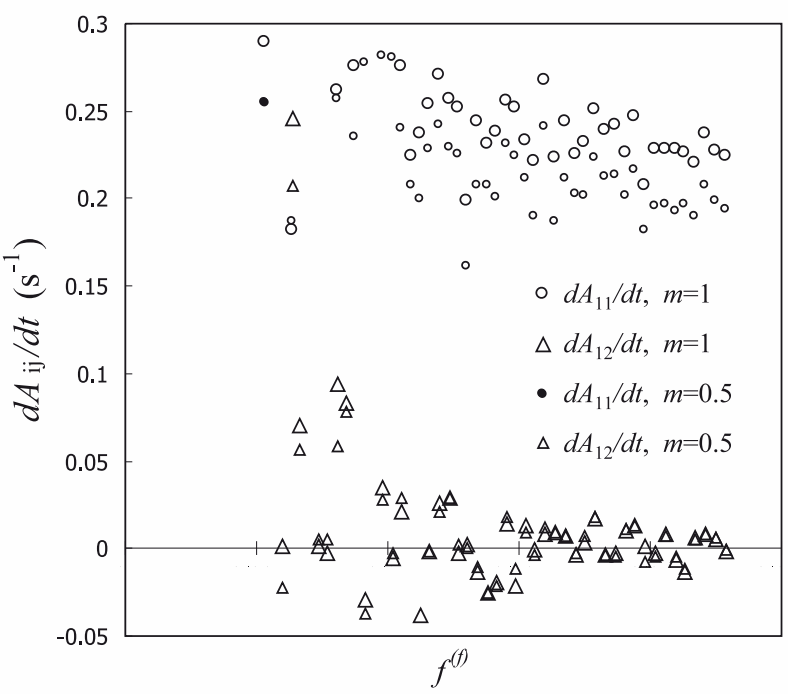

Figure 4. Influence de la fraction de fibres sur les contraintes $(a)$ et la vitesse d'orientation $(b)$, pour $m=1$ et $m=0.5$ - réseaux orientés selon $e_{1}\left(\widetilde{A}_{11} \approx 0.8\right)$

\subsection{Influence de l'orientation courante des fibres}

Dans la figure 5, nous présentons ensuite des résultats de compression en canal réalisés sur des réseaux à même fraction de fibres $\left(f^{(f)}=0.188\right)$, mais avec des orientations différentes, allant du cas parfaitement orienté dans la direction $\boldsymbol{e}_{2}$ $\left(\widetilde{A}_{11} \approx 0\right.$ ) jusqu'au cas parfaitement orienté dans la direction $\boldsymbol{e}_{1}\left(\widetilde{A}_{11} \approx 1\right)$. La figure $5(a)$ montre que les composantes du tenseur des contraintes $\widetilde{\sigma}_{i j}^{(f)}$ sont très sensibles à l'orientation courante (mesurée ici par $\widetilde{A}_{11}$ ), et ce pour les deux valeurs de $m$ envisagées. Le rapport des contraintes principales $\widetilde{\sigma}_{11}^{(f)} / \widetilde{\sigma}_{22}^{(f)}$ est voisin de 2.5 pour les orientations isotropes, alors qu'il tend vers des valeurs très fortes $(>50)$ pour les orientations prononcées selon $e_{1}$. De la même manière, la vitesse de réorientation des mèches dans la direction de la sollicitation (donnée par $\dot{\widetilde{A}}_{11}$ ) est elle aussi fortement influencée par l'orientation courante. Elle est maximale pour les microstructures quasi isotropes et décroît régulièrement avec l'intensité de l'orientation, que celle-ci soit dans la direction $\boldsymbol{e}_{1}$ ou $\boldsymbol{e}_{2}$. On peut remarquer que l'évolution de $\dot{\widetilde{A}}_{11}$ n'est pas exactement symétrique par rapport à $\widetilde{A}_{11}=0.5$, la décroissance vers zéro pour les valeurs de $\widetilde{A}_{11}$ faibles étant plus nette que pour les valeurs de $\widetilde{A}_{11}$ proches de 1 . Remarquons enfin que la composante $\dot{\widetilde{A}}_{12}$ n'est pas influencée par $\widetilde{A}_{11}$ (sollicitation dans les axes des microstructures). 
(a)

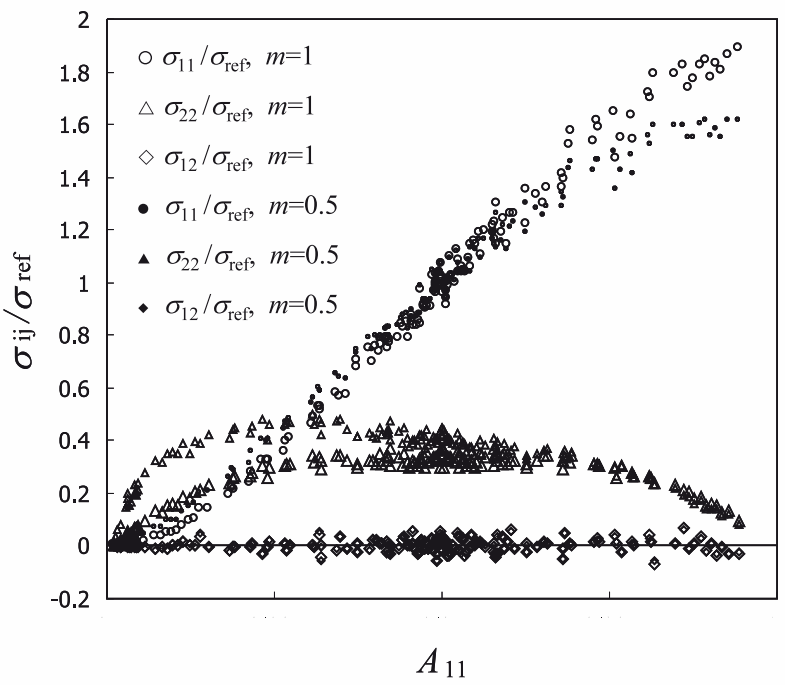

(b)

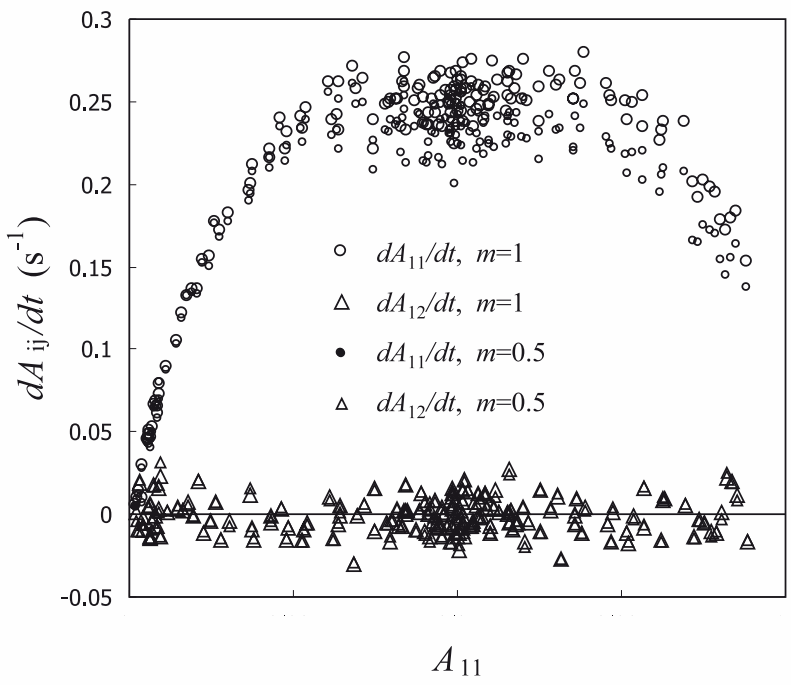

Figure 5. Influence de l'intensité de l'orientation $\left(\widetilde{A}_{11}\right)$ sur la réponse en contraintes (a) et vitesse d'orientation moyenne $(b)-f^{(f)}=0.188$, cellules orientées selon $\boldsymbol{e}_{1}$

\subsection{Influence de l'indice de sensibilité à la vitesse}

Sur les trois figures précédentes, nous avons représenté en parallèle les résultats du calcul d'homogénéisation pour deux valeurs de l'indice de sensibilité à la vitesse $m$. L'ensemble de ces figures tend à montrer que pour les deux valeur de $m$ utilisées, les contraintes normalisées ainsi que les taux d'orientation suivent quasiment les mêmes tendances à quelques nuances près qu'il faudrait confirmer par d'autres simulations :

- sur la figure $5(a)$, on note dans le cas $m=0.5$ une inflexion de la courbe $\widetilde{\sigma}_{11}^{(f)}\left(\widetilde{A}_{11}\right)$ pour $\widetilde{A}_{11}>0.75$ et une importante augmentation de $\widetilde{\sigma}_{22}^{(f)}$ pour $\widetilde{A}_{11}<0.3$,

- sur la figure $5(b)$ on remarque que pour $\widetilde{A}_{11}>0.3$, la composante $\dot{\widetilde{A}}_{11}$ est une fonction croissante de $m$.

\section{Conclusion}

Nous avons développé une approche numérique basée sur l'homogénéisation des structures discrètes périodiques pour analyser les relations entre la microstructure et les propriétés rhéologiques macroscopiques de suspensions de mèches de fibres concentrées avec une orientation plane. Le milieu est décrit comme un réseau de mèches indéformables dans le plan de la suspension, connectées par des liaisons visqueuses en loi puissance qui engendrent des forces et des moments d'interaction. Pour se rapprocher des cas industriels tels que ceux des SMC ou des TRE, nous avons mis 
en place une procédure de génération numérique pour construire des suspensions idéalisées avec différents taux de renfort et différentes orientations. Ceci nous a permis de réaliser des expériences de "rhéométrie numérique » mettant en évidence la forte influence de la microstructure sur le comportement macroscopique.

Le premier résultat de cette étude est théorique : si les interactions entre mèches suivent une loi puissance de la vitesse relative entre mèches, l'ensemble de la suspension se comporte également comme un fluide en loi puissance, avec la même sensibilité à la vitesse que celle postulée au niveau des interactions (Le Corre et al., 2004). De la même façon, la dérivée du tenseur d'orientation, et plus généralement la variation temporelle de l'orientation, est une fonction homogène de degré 1 par rapport au gradient de vitesse macroscopique (Le Corre et al., 2005). Ces résultats sont obtenus de manière théorique et ne dépendent pas de la technique de génération. De plus, la forme générale de la loi de comportement macroscopique montre que les contraintes sont des fonctions quadratiques de la fraction volumique de mèches : ceci est directement lié à la méthode de détection des contacts utilisée, qui suit le « modèle tube » statistique.

Il faut noter que ce modèle ne demande que très peu de paramètres rhéologiques, ceux-ci étant pour la plupart liés à des grandeurs physiques mesurables, associées à la morphologie des mèches de fibres. Malgré les hypothèses de modélisation très simples adoptées, les comparaisons avec des résultats expérimentaux sur des SMC (Le Corre et al., 2005) montrent une bonne corrélation pour les niveaux de viscosité dans les gammes de fractions de fibres et de vitesses de déformation testées. Les résultats numériques soulignent également les forts couplages entre l'orientation, le taux de fibres et la rhéologie de la suspension. Ils doivent être complétés par d'autres sollicitations mécaniques et d'autres paramètres microstructuraux. Ceci permettrait de constituer une base de données précieuse qui pourrait être comparée aux modèles rhéologiques existants, développés dans le cadre des suspensions semi-diluées et concentrées, ainsi qu'aux modèles décrivant l'évolution de l'orientation des fibres dans les suspensions.

\section{Remerciements}

Les auteurs souhaitent remercier la Région Rhône-Alpes, et leurs partenaires industriels Groupe Mecelec-Inoplast, Renault, Schneider Electric et Renault Trucks, pour leur soutien technique et financier.

\section{Bibliographie}

Advani S. G., Tucker C. L., " The use of tensors to describe and predict fiber orientation in short fiber composites », J. Rheol., vol. 3, n 8, p. 751-784, 1987.

Batchelor G., « The Stress System in a Suspension of Force-Free Particles », J. Fluid Mech., vol. 41, p. 545-570, 1970. 
Dumont P., Orgéas L., Le Corre S., Favier D., « Anisotropic Viscous Behaviour of Sheet Molding Compounds (SMC) During Compression Molding », Int. J. Plasticity, vol. 19, n 4, p. $625-646,2003$.

Dupret F., Verleye V., Modelling the Flow of Fiber Suspensions in Narrow Gaps, Rheology Series, Elsevier, Amsterdam, p. 1347-1398, 1999.

Ericsson K. A., Toll S., Månson J.-A. E., « Sliding Plate Rheometry of Planar Oriented Concentrated Fiber Suspension », Rheol. Acta, vol. 36, p. 397-405, 1997.

Folgar F., Tucker C., Lee C., « Simulation of compression molding for fiber-reinforced thermosetting polymers », J. Eng. Ind. - Trans. ASME, vol. 106, p. 114-125, 1984.

Gibson A., Toll S., « Mechanics of the squeeze flow of planar fibre suspensions », J. Non-Newt. Fluid Mech., vol. 82, p. 1-24, 1999.

Goddard J. D., « Tensile Stress Contribution of Flow-Oriented Slender Particles in NonNewtonian Fluids », J. Non-Newt. Fluid Mech., vol. 1, p. 1-17, 1976.

Jeffery G. B., « The motion of ellipsoidal particles immersed in a viscous fluid », Proc. Roy. Soc. London, vol. (A) 102, p. 161-179, 1922.

Le Corre S., Caillerie D., Orgéas L., Favier D., « Behavior of a Net of Fibers Linked by Viscous Interactions : Theory and Mechanical Properties », J. Mech. Phys. Solids, vol. 52, p. 395$421,2004$.

Le Corre S., Dumont P., Orgéas L., Favier D., « Rheology of highly concentrated planar fiber suspensions », J. Rheol., 2005. (accepted).

Le Corre S., Orgéas L., Favier D., Tourabi A., Maazouz A., Venet C., « Shear and compression behaviour of Sheet Molding Compounds », Compos. Sci. Technol., vol. 62, n 4, p. 571-577, 2002.

Mackaplow M. B., Shaqfeh E. S. G., « A Numerical Study of the Rheological Properties of Suspensions of Rigid, Non-Brownian Fibres », J. Fluid Mech., vol. 329, p. 155-186, 1996.

Moreau G., Caillerie D., « Continuum Modeling of Lattice Structures in Large Displacement Applications to Buckling Analysis », Comput. Struct., vol. 68, p. 181-189, 1998.

Petrich M. P., Koch D. L., « Interactions between contacting fibers », Phys. Fluids, vol. 10, n 8 , p. 2111-2113, 1998.

Ranganathan S., Advani S. G., « Fiber-Fiber Interactions in Homogeneous Flows of Non-Dilute Suspensions », J. Rheol., vol. 35, p. 1499-1522, 1991.

Servais C., Luciani A., Månson J.-A. E., « Fiber-fiber interaction in concentrated suspensions : dispersed fiber bundles », J. Rheol., vol. 43, n 4, p. 1005-1018, 1999a.

Servais C., Luciani A., Månson J.-A. E., « Squeeze flow of concentrated long fibre suspensions : experiments and model », J. Non-Newt. Fluid Mech., vol. 104, p. 165-184, 2002.

Servais C., Månson J.-A. E., Toll S., « Fiber-fiber interaction in concentrated suspensions : disperse fibers. ", J. Rheol., vol. 43, n 4, p. 991-1004, 1999b.

Toll S., « Note : On the Tube Model for Fiber Suspensions », J. Rheol., vol. 37, n 1, p. 123-125, 1993.

Toll S., Månson J.-A. E., « Dynamics of a Planar Concentrated Suspension with NonHydrodynamic Interaction », J. Rheol., vol. 38, n 4, p. 985-997, 1994.

Tollenaere H., Caillerie D., « Continuous modeling of lattice structures by homogenization », Adv. Eng. Softw., special issue, vol. 29, n 7, p. 699-705, 1998. 